Introduction According to Uganda Revenue Authority, there has been an increase of newly registered vehicles from $1.8 \%$ $38.7 \%$ from 2009 to 2013 . This has led to the emergence of many petrol stations in the country. However, there is little attention put in terms of safety and health especially in emergency preparedness and response. There is increasing evidence that emergency preparedness and response is one of the critical factors for improving safety and health at work places. Despite its importance, there seems to be little emphasis in its development and implementation in Uganda. We carried out routine inspections in the districts of Bushenyi and Sheema to assess emergency preparedness and response compliance with Uganda's Occupational Safety and Health Act 2006. This study was focused mainly in the area of fire safety and the presence fire assembly point.

Methods This study was carried out using Uganda's Occupational Safety and Health check list which was administered to 25 selected workplaces in the above districts from April 2015 up to April 2016. The data was collected and analysed using Excel programme.

Results Out of twenty six workplaces inspected, $69 \%$ had fire extinguishers, 31\% didn't have fire extinguishers, $66 \%$ had sand buckets and $34 \%$ had no sand buckets. All the twenty six workplaces had no fire alarm, no emergency action plan, no assembly point, no emergency contacts, no signage and no fire drills have ever been done.

Discussion The findings from this study show that emergency preparedness and response has not been paid much attention yet it is one of the strategies for improving safety and health at workplaces. The results also show the employers only knew fire extinguishers and sand buckets when it comes to the area of emergency preparedness and response. The fire extinguishers in some of these workplaces had long expired and were written in Chinese language which the locals could not comprehend. There is need to sensitise the employers and employees on the importance of developing and implementing a sound emergency preparedness and response plan.

\section{HUMAN BIOMONITORING FOR EMERGENCY RESPONDERS - EXPERIENCE, BENEFITS AND LIMITATIONS -}

${ }^{1} \mathrm{M}$ Bader, ${ }^{1} \mathrm{~S}$ Bäcker, ${ }^{1} \mathrm{~T}$ Jäger, ${ }^{2} \mathrm{G}$ Van Bortel, ${ }^{3} \mathrm{~T}$ van Weyenbergh, ${ }^{3} \mathrm{E}$ Verwerft, ${ }^{1} S$ Webendörfer, ${ }^{1} \mathrm{C}$ Oberlinner, 'S Lang. 'BASF SE, Corporate Health Management, Ludwigshafen, Germany; ${ }^{2} B A S F$ SE, Emergency Response, Ludwigshafen, Germany; ${ }^{3} B A S F$ Antwerpen N.V., Occupational Medicine and Health Protection, Antwerp, Belgium

\subsection{6/oemed-2018-ICOHabstracts.330}

Introduction Human Biomonitoring (HBM) was introduced decades ago for the monitoring of workers' exposure to chemicals. Meanwhile, procedures like sample collection/transport are standardised and assessment values are continuously derived or updated. But HBM is also a useful tool for monitoring exposure after incidents such as chemical spills or large fires. Accordingly, the exposure situation of firefighters, other emergency responders or bystanders has come into the focus of occupational and environmental medicine and toxicology.

Methods A standardised and streamlined procedure for the HBM of emergency responders was established at two large chemical production sites. The key constituents of the program comprise a tick box list of altogether 36 chemicals, a short questionnaire, and a predefined sampling/transport chain. In 2016, this program was carried out after a major accident on one site. Samples were collected from firefighters, other company employees and external rescue forces. Exposure to benzene, toluene and polycyclic aromatic hydrocarbons (PAHs) was monitored using sensitive, specific and quality-controlled biomonitoring methods.

Results An additional exposure to benzene was found only in firefighters, with a maximum S-Phenylmercapturic Acid (SPMA) concentration of about $40 \mu \mathrm{g} / \mathrm{g}$ creatinine. Most results were in the range of the general background, but moderately increased into the typical range of smokers. Benzene exposure was not only confirmed for active firefighters but also for post-accident firewatch forces. Biomarkers for toluene and PAHs were all within the normal range, which was unexpected since PAH biomarkers are often found in post-operation samples of firefighters. However, this result accords with the observation of an almost vertical smoke propagation.

Conclusion A high degree of preparedness is essential for the conduction of HBM programs after chemical incidents. Professional firefighters are usually well prepared to avoid overexposure to chemicals. However, residual spot contamination can be relevant and needs to be considered for professional safety measures.

\section{INTEGRATING OCCUPATIONAL HEALTH \& SAFETY THROUGH EFFECTIVE OCCUPATIONAL HEALTH PROGRAM}

Sandeep Sharma*, SK Awasthi. IndiaOil Corporation Ltd, New Delhi, India

\subsection{6/oemed-2018-ICOHabstracts.331}

Introduction Indian Oil Corporation has taken a series of initiatives to ensure safe and healthy working conditions for employees. Aim of the study was to strengthen and streamline the Occupational Health and Emergency Medical Services across the Corporation.

Materials and methods Occupational Health Services (OHS) audit of Ten IndianOil Refinery units was conducted and following points were observed-Well First Aid Centre, Provision of dedicated communication facility, which can be activated in the event of fire/Accident in Refinery, Prominent display of Siren Protocol for Accident/Disaster, Drug register,System of daily check of emergency drugs, oxygen cylinders and other resuscitation equipment, daily check of the refrigerator, Proper record of illnesses and injuries, updated record of 'at-risk' hazardous materials used or produced at the worksite, Updated version of Material Safety Data Sheets-for acute exposure management, Record of daily check of housekeeping, Provision and maintenance of First aid boxes equipped with prescribed content, readily accessible during all working hours, Percentage of employees identified for FA training, Well equipped Ambulance van should be available for the purpose of transportation of serious cases of accidents or sickness, Training in BLS (Basic Life Support)(CPR-Cardio Pulmonary Resuscitation),Disaster Management Plan (DMP),Emergency preparedness plan Functional organogram in case of disaster situation Details of facilities available at nearby hospitals, are Triage guidelines incorporated and training imparted to employees.

Occupational Health and Wellness Index was created and implemented across the Corporation to further reinforce these targeted efforts.

Result The purpose of the study for establishing Occupational Health and Emergency Medical Services management frame- 